\title{
Denuder Tube Preconcentration and Detection of Gaseous Ammonia Using a Coated Quartz Piezoelectric Crystal
}

\author{
Zulfiqur Ali, C. L. Paul Thomas and John F. Alder* \\ Department of Instrumentation and Analytical Science, UMIST, P.O. Box 88, Manchester M60 1QD, UK \\ Geoffrey B. Marshallt \\ National Power Technology and Environmental Centre, Kelvin Avenue, Leatherhead, Surrey KT22 7SE, \\ UK
}

\begin{abstract}
The feasibility of using a cylindrical denuder tube for sampling gaseous ammonia, followed by detection with a piezoelectric quartz crystal, was investigated. Gaseous ammonia was sampled with a tungsten oxide-coated cylindrical denuder tube and then thermally desorbed onto a piezoelectric quartz crystal coated with pyridoxine hydrochloride-Antarox CO-880. A linear calibration graph of peak area response versus ammonia concentration sampled was obtained for ammonia concentrations between 3.1 and $8.2 \mu \mathrm{gl}^{-1}$. A concentration of $29 \mathrm{ng} \mathrm{I}^{-1}$ of ammonia in air was detected with a signal-to-background ratio of $14: 1$ by achieving an enrichment ratio of 900 with the tungsten oxide denuder tube.
\end{abstract}

Keywords: Ammonia; piezoelectric crystal; denuder tube; preconcentration

Ammonia is one of the most important trace gases in the atmosphere and is the only one that is basic. It is water-soluble and can react with aerosols, thus influencing atmospheric acidity. Most ammonia emissions are released into the atmosphere by biological processes, primarily through the decomposition of organic matter. ${ }^{1}$ The main industrial source is from fertilizer and ammonia production plants. ${ }^{2}$

In determining the precise role of ammonia in the atmosphere, it is important to distinguish free ammonia from ammonium particulates. Filtration techniques have been used for separation of the gaseous phase from particulates, although their use can cause errors by the introduction of artefacts. For instance, an over-estimation of the ammonia concentration can be obtained by release of ammonia from ammonium nitrate on the filter-paper. Equally, an underestimation can be obtained by reaction of gaseous ammonia with acids deposited on the filter.

Denuder tubes have been shown to be effective for the separation of gases from particles, and their theory and application for the determination of gaseous species have been reviewed. ${ }^{3.4}$ Air is drawn under conditions of laminar flow through a tube coated with a selective adsorbent. Gaseous species diffuse to the collection surfaces. Particulates, having much lower diffusion velocities, cannot migrate to the walls and hence pass through unabsorbed and do not contribute to the final measurement. Gormley and Kennedy ${ }^{5}$ have derived a solution describing diffusion from a stream flowing through a cylindrical tube:

$$
\begin{aligned}
\frac{c}{c_{0}}=0.819 \exp (14.6272 \Delta)+ & 0.0976 \exp (-82.22 \Delta) \\
& +0.01896 \exp (-212 \Delta)
\end{aligned}
$$

where $c$ is the mean concentration of gas leaving the tube, and $c_{1}$ is the gas concentration entering the tube.

$$
\Delta=\frac{\pi D l}{4 F}
$$

where $D$ is the diffusion coefficient of the target gas in air, $l$ is the length of coated tube, and $F$ is the flow rate.

For $\Delta \geqslant 0.05$ only the first term in eq. (1) is significant.

$$
c \simeq 0.819 c_{0} \exp (-14.6272 \Delta)
$$

\footnotetext{
* To whom correspondence should be addressed.

† Present address: Chemistry Department, Birkbeck College, 20 Gordon Square. London WC1H OPP, UK.
}

The gas flow is, therefore, depleted by diffusion of the gaseous component to the walls, where it is adsorbed. The particulate phase, being heavier, passes through the tube.

An oxalic acid-coated denuder tube has been used for the determination of free ammonia in ambient air. ${ }^{6,7}$ The method described requires washing the sorbed ammonia from the tube and determining it by potentiometry with an ammonia-sensitive probe. A tungsten oxide-coated denuder tube has also been used for sampling ammonia, which was then thermally desorbed and determined by means of a chemiluminescence nitrogen detector. ${ }^{8}$ Hlavay and Guilbault ${ }^{9}$ have used a pyridoxine hydrochloride-coated piezoelectric quartz crystal for the detection of ammonia. Pyridoxine hydrochloride reversibly adds ammonia to the phenolic $3-\mathrm{OH}$ :

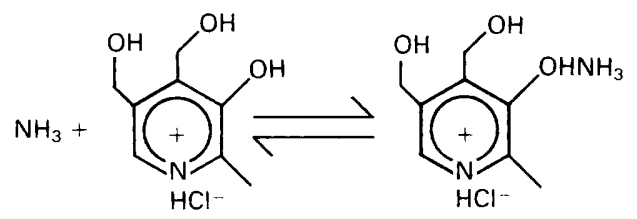

A reported frequency change of $1190 \mathrm{~Hz}$ for $1 \mathrm{ppm}$ of ammonia in air was obtained, while Moody et al. ${ }^{10}$ extended the useful lifetime of the detector by supporting the pyridoxine hydrochloride in a nonyl phenoxypolyethoxylate matrix of high relative molecular mass (Antarox CO-880). This was, however, at the expense of decreased sensitivity. Antarox CO-880 did not itself adsorb ammonia.

The present paper describes the application of a tungsten oxide-coated denuder tube to the collection of gaseous ammonia, which is subsequently thermally desorbed and detected by a pyridoxine hydrochloride-Antarox CO-880 coated, $14.9 \mathrm{MHz}$ piezoelectric quartz crystal. The frequency responses of the piezoelectric quartz crystal detector to various concentrations of ammonia were measured.

\section{Experimental}

\section{Piezoelectric Quartz Crystal Oscillator}

The piezoelectric quartz crystals used were $14.9 \mathrm{MHz}$, fundamental mode, AT-cut with $3.8 \mathrm{~mm}$ diameter circular gold electrodes, on both sides of the $8 \mathrm{~mm}$ diameter quartz slab, supplied by Cathodeon Crystals, Cambridge, UK. The reference and detector crystals were made part of two pre-fabricated crystal oscillator circuits (Cathodeon Crystal) 
powered from a $5 \mathrm{~V}$ d.c. power supply. The oscillator outputs were mixed in the exclusive-OR gates of a 74LS86 operational amplifier (RS Components, Corby, Northamptonshire, UK), and a low-pass filter was used to select the difference frequency, which was passed to a $120 \mathrm{MHz}$ range, $0.01 \mathrm{~Hz}$ resolution digital frequency counter (Philips PM6671; Philips, Cambridge, UK), equipped with a digital-to-analogue converter. The sensor crystal was housed in a double-impinger type cell with a swept volume of $0.7 \mathrm{ml}$, sample gas being injected perpendicular to each face of the coated crystal.

\section{Ammonia Determination}

A continuous-gas flow regime was used for the determination of ammonia. Cylinder air (dried over silica gel) was used as the carrier gas. Poly (tetrafluoroethylene) (PTFE) tubing $(6.5 \mathrm{~mm}$ o.d., Omnifit, Cambridge, UK) was used for all supply and waste lines. Gas flows were controlled by needle valves and were measured by rotameters calibrated against a soap-film bubble meter.

Low concentrations of ammonia in air were produced by two-stage dilution of a $100 \mathrm{ppm}$ ammonia-in-air standard mixture (BOC Special Gases, London, UK). A four-way valve (Omnifit) was used to switch from the reference air flow to the sampling flow. A flow rate of $20 \mathrm{ml} \mathrm{min}^{-1}$ was maintained in the piezoelectric quartz crystal detector cell by means of a needle valve.

Pyridoxine hydrochloride-Antarox CO-880 coated piezoelectric quartz crystals were prepared by brush-coating a $1+1(\mathrm{v} / \mathrm{v})$ mixture of $0.02 \% \mathrm{~m} / \mathrm{v}$ pyridoxine hydrochloride in $50 \% \mathrm{v} / \mathrm{v}$ aqueous ethanol and a $0.2 \% \mathrm{~m} / \mathrm{v}$ solution of Antarox CO-880 in acetone. The coated quartz crystals were then kept in an oven at $80^{\circ} \mathrm{C}$ for $2 \mathrm{~h}$ and allowed to cool in a desiccator.

\section{Preparation of Tungsten Oxide Denuder Tube}

Quartz tubes were coated with tungsten oxide by using a modification of the procedure described by Braman et al ${ }^{8}$ The quartz tubing ( $3 \mathrm{~mm}$ i.d., $5 \mathrm{~mm}$ o.d., $35 \mathrm{~cm}$ length) was prepared for coating by first washing with benzene and then with $50 \%$ sodium hydroxide solution. The tubes were then treated with $40 \% \mathrm{~m} / \mathrm{v}$ hydrofluoric acid before being finally rinsed with high-purity water and dried in an oven at $150^{\circ} \mathrm{C}$ for $2 \mathrm{~h}$. Flow rates of less than $11 \mathrm{~min}^{-1}$ were used for much of the gas sampling work. A $10 \mathrm{~cm}$ subduction zone was made by pipetting a solution of trimethylsilane in dichloromethane to the $10 \mathrm{~cm}$ mark, draining and then washing with high-purity water and drying at $150^{\circ} \mathrm{C}$ for $2 \mathrm{~h}$.

The denuder tubes were coated by means of the apparatus shown in Fig. 1. The quartz tube to be coated was connected between two Swagelok T unions with $0.25-0.125$ in reducing PTFE ferrules. Tungsten wire $(0.5 \mathrm{~mm}$ o.d.; Goodfellow Metals, Cambridge, UK) was spot-welded to stainless-steel rods $(0.125$ in o.d.) at each end. Several welds were necessary to achieve a mechanically strong join between the wire and rod. The stainless-steel rods and tungsten wire were threaded through the denuder tube and the $T$ union compression fittings. A gas-tight seal was formed by using the PTFE reducing ferrule (Phase Separations, Deeside Industrial Estate, Clwyd, UK).

The denuder tube was evacuated to $267-400 \mathrm{~Pa}$ with a rotary vacuum pump. The tungsten wire was heated to approximately $1000^{\circ} \mathrm{C}$ by passing through it a current (a.c.) of $12 \mathrm{~A}$. Heating the tungsten wire caused expansion, and the tension was maintained by carefully pulling the stainless-steel rods with insulated pliers. The current was controlled by a variable transformer to provide a slow coating rate. A light coating of the blue oxide was obtained after passage of $12 \mathrm{~A}$ for $30 \mathrm{~min}$; a heavier coating was obtained after $2 \mathrm{~h}$. Denuder tubes coated rapidly at higher currents resulted in mechanically less stable coatings. The blue tungsten(Iv) oxide was obtained by heating the tube to $350^{\circ} \mathrm{C}$ at a pressure of approximately $650 \mathrm{~Pa}$. The denuder tube was heated with a Kanthal resistance wire heater (Scientific Wire Co., London, UK). The resistance wire was wrapped around the quartz tube and held in place with hose clips at both ends. The temperature of the denuder tube was monitored by placing in the tube a thermocouple connected to two strands of Kanthal wire, with output to a digital thermometer.

\section{Chemiluminescence Nitrogen Detector}

A chemiluminescence nitrogen detector (Monitor Laboratories, Model $8440 \mathrm{E}$ ) was used to characterize the tungsten oxide denuder tubes. Operation of the detector was based on the chemiluminescence reaction between nitrogen oxide and ozone. The nitrogen oxide and ozone reaction yielded stable nitrogen dioxide, primarily in the electronic ground state. A small fraction of the reaction yielded excited nitrogen dioxide, which emitted an infrared/visible continuum with a maximum intensity at approximately $1.1 \mu \mathrm{m} .{ }^{11}$ Determination of ammonia was achieved by chemically converting the sample into nitrogen oxide in a stainless-steel converter at $850^{\circ} \mathrm{C}$, prior to its entry into the reaction cell. The instrument was calibrated against standard nitrogen oxide mixtures (BOC Special Gases) and calibrated for ammonia by passing standard ammonia-air mixture into the converter.

\section{Characterization of the Tungsten Oxide Denuder Tube}

The collection efficiency of the tungsten oxide denuder tube was established by measuring the decrease in the steady-state signal, when the denuder tube was inserted between the standard ammonia sample stream and the chemiluminescence nitrogen detector. Desorption of the adsorbed ammonia was carried out by heating the denuder tube from ambient temperature to $350^{\circ} \mathrm{C}$, with a desorption time of $1 \mathrm{~min}$. The recovery efficiency was measured by dividing the area under

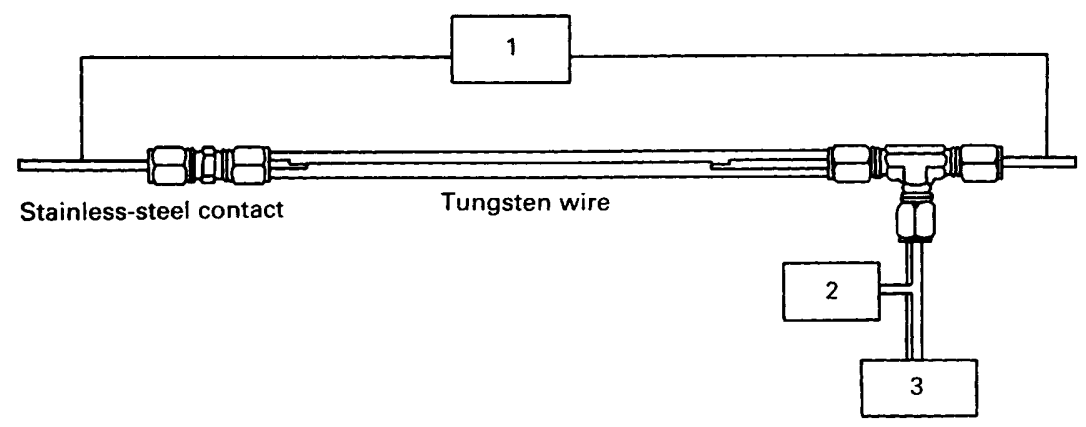

Fig. 1 Apparatus used for coating the denuder tubes with tungsten oxide. For details, see text. 1, A.c. voltage controller; 2, Pirani vacuum gauge; and 3 , rotary vacuum pump 


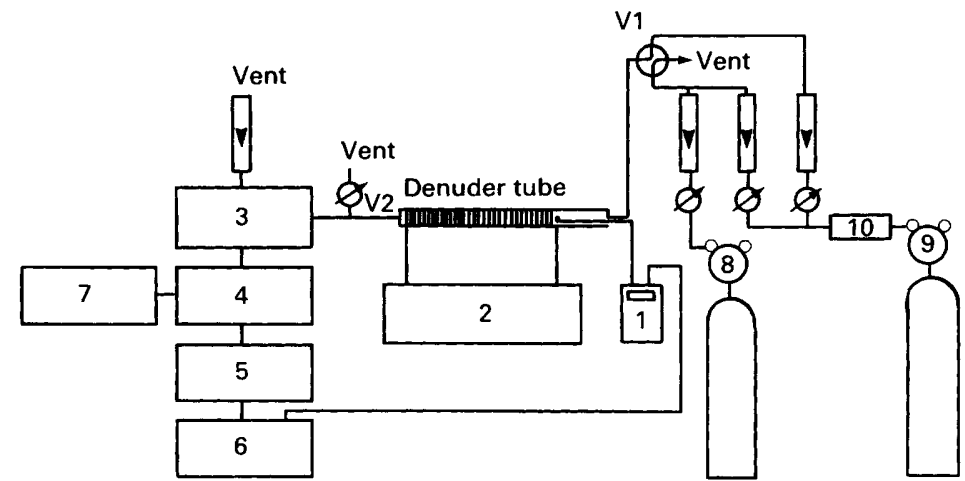

Fig. 2 Apparatus for the preconcentration of ammonia on the denuder tube, and subsequent desorption onto the piezoelectric crystal detector. 1, Digital thermometer; 2 , a.c. voltage controller; 3 , piezoelectric crystal detector cell; 4 , oscillator; 5 , frequency counter; 6 , chart recorder; 7 , power supply; $B$, ammonia, $100 \mathrm{ppm}$ in air; 9 , air; and 10 , drying tube

the thermal desorption peak by the area under the collection profile. This ratio corresponds to the mass of ammonia desorbed from the denuder tube divided by the mass sorbed from the air stream.

The linearity of response of the denuder system for ammonia detection was measured by introducing known mixtures of increasing ammonia concentration into the tungsten oxide denuder tube. Ammonia standards were prepared by dilution of standard $100 \mathrm{ppm}$ ammonia-in-air mixtures. The adsorption capacity of the denuder was ascertained by subjecting it to an ammonia concentration of 60 $\mu \mathrm{g} \mathrm{m}^{-3}$ in air at a flow rate of $100 \mathrm{ml} \mathrm{min}^{-1}$, noting the time taken for breakthrough to the $\mathrm{NO}_{x}$ detector.

\section{Sampling and Detection of Gaseous Ammonia by Denuder Tubes and Piezoelectric Quartz Crystals}

A schematic diagram of the system used to sample and detect gaseous ammonia is shown in Fig. 2. Low ammonia concentrations were generated from permeation tubes, prepared in the laboratory, having typical permeation rates of $180 \mathrm{ng} \mathrm{min}^{-1}$ at a temperature of $28^{\circ} \mathrm{C}$. Standard ammonia gas streams were routed to the tungsten oxide denuder tube through a four-way valve V1. The collected ammonia was thermally desorbed into a dry air stream. Detection of ammonia was carried out by an AT-cut $14.9 \mathrm{MHz}$ piezoelectric crystal coated with pyridoxine hydrochloride-Antarox CO-880.

\section{Results and Discussion}

The effect of temperature on the preparation of the denuder tube, and the desorption temperature, were examined. Tungsten oxide denuder tubes subjected to a desorption temperature of $500^{\circ} \mathrm{C}$ showed degradation in response with successive sampling cycles, using chemiluminescence nitrogen detection. Desorption products included nitrogen oxide, nitrogen dioxide and ammonia. Tungsten oxide denuder tubes subjected to temperatures of less than $350^{\circ} \mathrm{C}$ gave ammonia as the only desorption product, and 11 successive sampling and analysis cycles of a $3 \mathrm{ppm}$ ammonia standard gas stream resulted in peak heights with a relative standard deviation of $3 \%$. The collection and recovery efficiencies were found to be 97 and $96 \%$, respectively. The linearity of quantitative adsorption of ammonia by the tungsten oxide denuder tube was demonstrated over ammonia mass loadings of $0.2-3.3 \mu \mathrm{g}$, with a correlation coefficient of 0.998 . These results show that the tungsten oxide denuder tube is capable of reproducible and quantitative sampling of ammonia. Ammonia thermally desorbs at a temperature near to that where degradation of the denuder occurs. The reliability of the technique is, therefore, dependent on accurate temperature control.

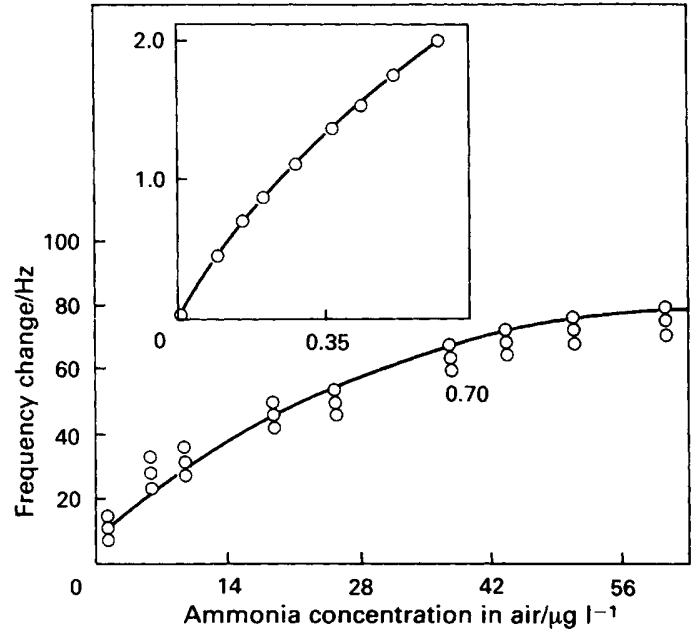

Fig. 3 Calibration graph for the coated piezoelectric crystal detector over the range $0.7-63 \mu \mathrm{g} \mathrm{I}^{-1}(1-90 \mathrm{ppm})$ of ammonia. The inset shows the calibration in the range $0-0.7 \mu \mathrm{g}^{-1}(0-1 \mathrm{ppm})$ of ammonia obtained at a different time

Fig. 3 shows the response curve for the direct exposure of the piezoelectric crystal to increasing concentrations of ammonia in dry air. The sensitivity obtained is lower than that reported by Hlavay and Guilbault. ${ }^{9} \mathrm{~A}$ linear change in frequency with sorbed mass is expected for low mass loadings on the piezoelectric crystal, ${ }^{12-15}$ hence the response curve will represent the ammonia sorption isotherm. The curve is similar to a Langmuir isotherm, and this is a reasonable model for a surface such as pyridoxine hydrochloride with clearly identifiable active sites.

Thermal desorption cycles of the tungsten oxide denuder tube, without added ammonia, resulted in frequency decreases of the sensor piezoelectric quartz crystal. The frequency decreases were thought to be as a result of an elevation in the temperature of the piezoelectric quartz crystal and expansion of the desorption gas volume. The effects could be decreased by increasing the desorption gas flow rate. A plot of this blank peak area versus desorption gas flow rate (Fig. 4) showed an exponential decrease of the peak with increasing desorption volume flow. A compromise high-desorption flow will, however, result in increased dilution of the desorbed analyte. A desorption flow rate of $100 \mathrm{ml} \mathrm{min-1,} \mathrm{which}$ minimized the peak, but maintained practical sensitivity, was used for all further studies.

The linearity of response of the piezoelectric quartz crystal denuder system for ammonia sampling and detection was tested by exposing the tungsten oxide denuder to ammonia concentrations between 3.1 and $8.2 \mu \mathrm{g} \mathrm{1}^{-1}$. A linear plot of 


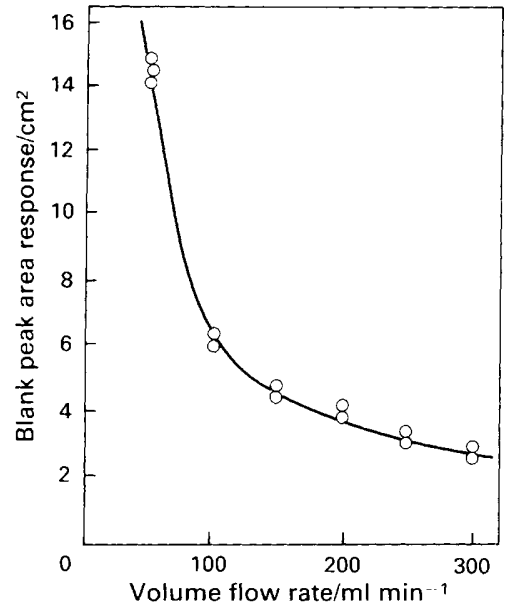

Fig. 4 Plot of coated piezoelectric crystal blank response peak area versus air flow rate through the denuder tube

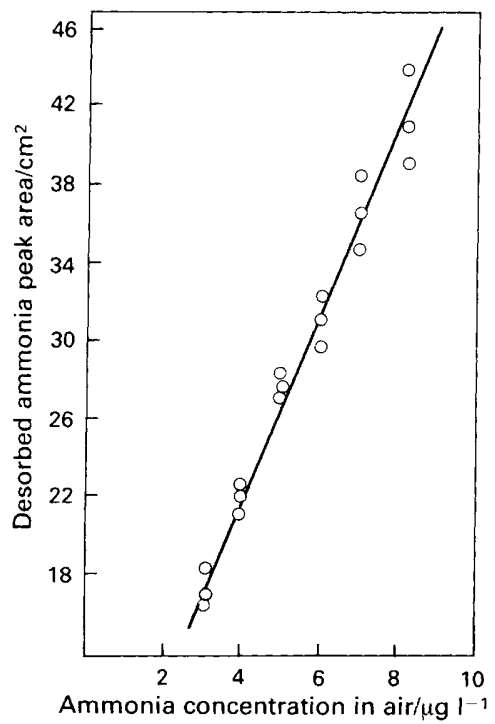

Fig. 5 Plot of ammonia response peak area from the coated piezoelectric crystal versus concentration of ammonia sampled in the denuder tube. The correlation coefficient of the straight line is 0.97 ; the ordinate axis intercept is 7.1

peak area response versus concentration of ammonia is shown in Fig. 5. The linearity of response of the piezoelectric quartz crystal/denuder system was further tested by varying the exposure times of a $6.1 \mu \mathrm{g} \mathrm{I}^{-1}$ ammonia gas stream to a tungsten oxide denuder tube. Exposure times of between 30 and $210 \mathrm{~s}$ resulted in $0.3-2.1 \mu \mathrm{g}$ calculated ammonia mass loadings. A least-squares fit analysis of the peak area response versus calculated mass loading yielded a straight line between 0.6 and $2.1 \mu \mathrm{g}$, with a slope of $25 \mathrm{~cm}^{2} \mu \mathrm{g}^{-1}$ and with a $y$-axis intercept of $-2.0 \mathrm{~cm}^{2} \mu \mathrm{g}^{-1}$. The correlation coefficient for the straight line was 0.99 . Below $1 \mu \mathrm{g}$ of ammonia, the line curved towards the origin. The adsorption capacity was calculated to be $2.9 \mu \mathrm{g}$ of ammonia, which compares favourably with a value of $2.5 \mu \mathrm{g}$ in earlier work ${ }^{16}$, when using the same method, and with $3 \mu \mathrm{g}$ from breakthrough studies.

Ammonia thermal-desorption profiles show the initial blank response followed by the ammonia peak. The tailing of the analyte peak increased with the mass of ammonia exposed. Such tailing could be due to a number of factors. The collection surface of the tungsten oxide denuder tube is not homogeneous, and the adsorption sites will have a range of binding energies, resulting in tailing of the desorption peak.

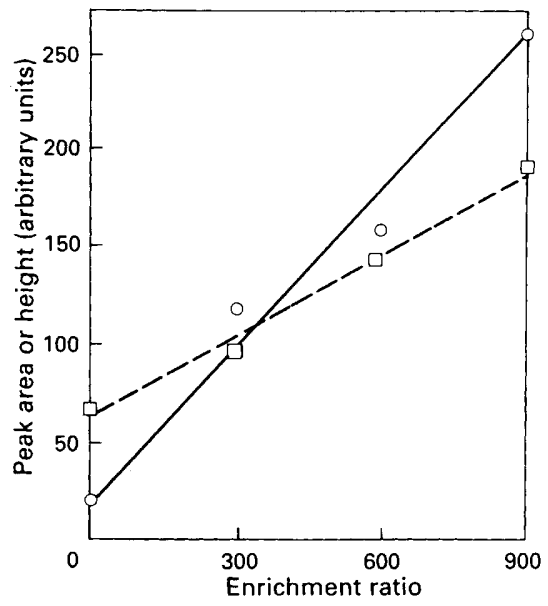

Fig. 6 Plots of peak height $(\square)$ and peak area $(\bigcirc)$ for the coated piezoelectric crystal response versus enrichment ratio calculated for the tungsten oxide-coated denuder tube

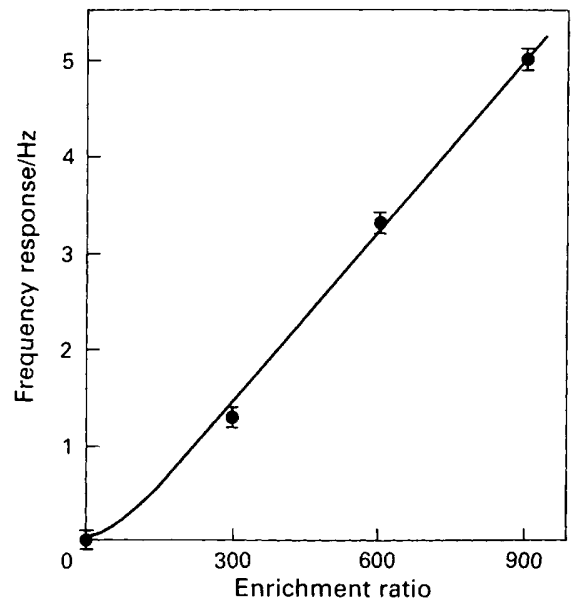

Fig. 7 Plot of frequency response versus enrichment ratio

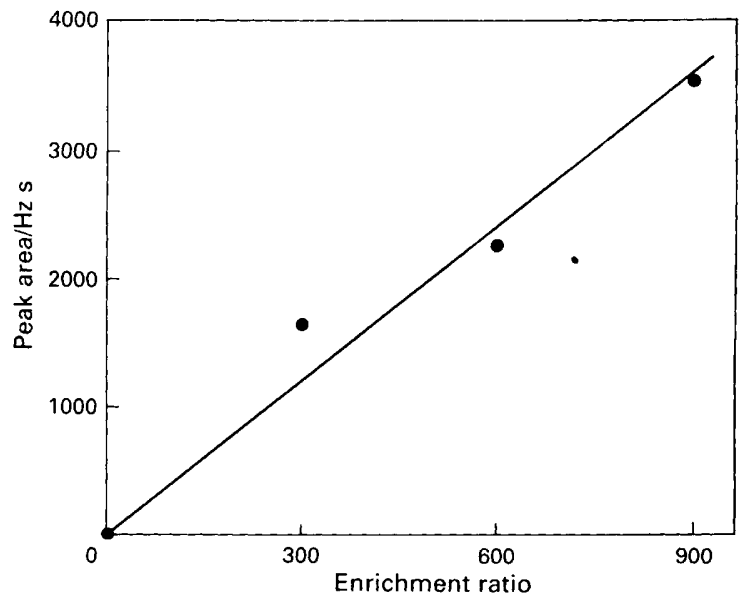

Fig. 8 Plot of integrated peak area versus enrichment ratio

Diffusional broadening and adsorptive retardation by the sampling lines and fittings could also lead to tailing.

\section{Preconcentration of the Analyte}

The denuder tube, in addition to separating gaseous ammonia from particulates, also serves to preconcentrate the analyte and hence increase the sensitivity of the system. The extent of 
preconcentration is determined by the enrichment ratio (ER), defined as the ratio of the concentration of ammonia in the desorbed gas $\left(c_{\mathrm{D}}\right)$ to that in the ambient air $\left[c_{0}\right.$; see eqn. (3)]. If the denuder tube preconcentrator is exposed until it is saturated, a significant part of the sample can be lost, but the ER is optimized. If the sampling time is restricted such that breakthrough does not occur, higher sampling efficiency, but lower enrichment ratios, are observed.

A study was carried out to illustrate the improvement in sensitivity of the system with an increase in the enrichment ratio. An ammonia-in-air concentration of $29 \mathrm{ng} \mathrm{l}^{-1}$ was passed over the tungsten oxide denuder tube for times sufficient to yield enrichment ratios of 300,600 and 900 . The data obtained are shown in Figs. $6-8$ as plots of peak frequency response and integrated area response for the blanks and three enrichment ratios. It is clear that this procedure significantly enhances the over-all sensitivity of the determination.

\section{Conclusions}

The feasibility of using a tungsten oxide denuder tube preconcentrator with a pyridoxine hydrochloride-Antarox CO-880 coated piezoelectric quartz crystal for the sampling and detection of ammonia has been demonstrated. Analysis for low concentrations of ammonia can be carried out if high enrichment ratios are used. The enrichment ratios could be further increased by increasing the adsorption capacity of the denuder tubes by using an annular denuder, which would also permit much shorter sampling times. The use of denuder preconcentrators thus leads to the significant advantage of being able to desorb the sampled vapour in dry carrier gas, hence overcoming the effect of relative humidity on the piezoelectric crystal. Although the temperature pulse has an effect on the piezoelectric crystal, it can be minimized and useful sensitivity can be achieved. The data in Figs. 6-8 represent a signal-to-background ratio of about $14: 1$ in integrated area terms, with an enrichment ratio of 900 for an ammonia concentration of $29 \mathrm{ng} \mathrm{l}^{-1}$, which represents a limit of detection of the order of $6.5 \mathrm{ng}^{-1}$ of ammonia.

The effect of interfering species on this technique has not been studied in this work. Previously published data indicate that alkylamines, nitrogen dioxide, peroxyacetyl nitrate and nitric acid adsorb into denuder tubes with tungsten(vi) oxide coatings. ${ }^{3}$ However, none of these species in the gas phase has been reported as adsorbing onto coatings of pyridoxine hydrochloride, and hence the second-stage selectivity of the piezoelectric crystal detector should minimize their action. It is also expected that aerosol and particulate interference will be minimal.

The authors are grateful to SERC and the then CEGB for support of Z. A. under the CASE scheme. The contribution of G. B. M. is published by permission of National Power plc.

\section{References}

1 Bujisman, E., Moss, H. F., and Asman, W. A. H. Atmos. Environ., 1987, 21, 1009.

2 Dawson, G. A., J. Geophys. Res., 1977, 82, 3125.

3 Ali, Z., Thomas, C. L. P., and Alder, J. F., Analyst, 1989, 114, 759.

4 Murphy, D. M., and Faheg, D. W., Anal. Chem., 1987, 59, 2753.

5 Gormley, P. G., and Kennedy, M., Proc. R. Ir. Acad., Sect. A., 1949, 52A, 103.

6 Ferm. M. Atmos. Environ., 1979, 13, 1385

7 Dimmock, N. A., and Marshall, G. B., Anal. Chim. Acta, 1986, 185, 159.

8 Braman, R. S., Shelley, T. J., and McClenny, W. A., Anal. Chem., 1982, 54, 365.

9 Hlavay, J., and Guilbault, G. G., Anal. Chem., 1978, 50, 1044.

10 Moody, G. J., Thomas, J. D. R., and Yarmo, M. A., Anal. Chim. Acta, 1983, 155, 225

11 Clyne, M. A. A., Thrush, B. A., and Wayne, R. P., Discuss. Furaday Soc., 1964, 60, 359.

12 Sauerbrey, G. Z., Z. Phys. Chem. (Leipzig), 1959, 155, 206.

13 Miller, J. G., and Bolef. D. I., J. Appl. Phys., 1968, 139, 5815.

14 Lu, C. S., J. Vac. Sci. Technol., 1975, 12. 578.

15 Cumpson, P. J., and Seah, M. P., Meas. Sci. Technol., 1990, 1. 544.

16 Ali, Z., PhD Thesis, UMIST, University of Manchester, 1989.

Paper 0/05024E

Received May 23, 1991

Accepted November 5, 1991 